\title{
Programa de intermediación laboral para jóvenes que han infringido la ley, la experiencia de Fundación Proyecto B
}

\section{Proyecto B: A work reentry program for juvenile offenders}

María Eugenia Sosa

Investigadora social, Magíster (c) en Ciencias Sociales, Mención Estudios de la Sociedad Civil. Encargada de Estudios, Proyecto B.

\begin{abstract}
Resumen
Desde la implementación de la Ley de Responsabilidad Adolescente (LRPA) en el año 2007, la población penal juvenil en Chile ha decantado en un "envejecimiento" de los/las jóvenes que cumplen sanción en el Servicio Nacional de Menores, para quienes muchas veces resulta insuficiente la intervención con enfoque educacional que norma dicha ley. Ante ello Fundación Proyecto B ha generado un programa basado en la intermediación laboral que busca aumentar la empleabilidad de los/las jóvenes participantes y contribuir a su proceso de reinserción social. El presente artículo busca presentar los avances en cuanto a diseño y manualización del programa, así como describir sus principales resultados. A diciembre del año 2013 el programa se habia aplicado a 187 jóvenes y presenta una tasa de reincidencia posdesvinculación del programa del $17,5 \%$, cifra que si bien no es concluyente debido a limitaciones metodológicas, es un punto de partida para una eventual medición de impacto.
\end{abstract}

Palabras claves. Jóvenes infractores de ley, reinserción social, empleabilidad.

\begin{abstract}
The Chilean juvenile population's evolution has shown a slow but steady aging tendency over the past decade. An aging juvenile population for whom the education-centered juvenile justice system interventions, carried on by the Servicio Nacional de Menores, sometimes falls short. In response to this Fundación Proyecto $B$ has designed an employability program aimed to promote and contribute to its participant's social reentry.

Throughout this article the program will be presented, as well as the main results obtained since its implementation. By December 2013 the program had delivered services to 187 youths and presents a post-participation recidivism rate of $17.5 \%$. This recidivism rate, even if it's not conclusive due to the limitations of the study, it's considered a starting point to a future impact evaluation.
\end{abstract}

Key words. Young offenders, social reentry, employability

\section{Introducción}

La seguridad ciudadana se ha mantenido durante las últimas décadas dentro de las principales preocupaciones de la población chilena y del gobierno, generándose una serie de modificaciones en la política criminal orientadas a disminuir los niveles de delincuencia en el país (Fundación Paz Ciudadana, 2014).
En este marco, y desde el año 2007, tras la entrada en vigencia de la Ley $N^{\circ} 20.084$ de Responsabilidad Penal Adolescente (LRPA) se ha puesto en marcha un sistema de respuesta ante la problemática específica de la delincuencia juvenil, la cual si bien representa un porcentaje menor en relación con la criminalidad adulta, sería responsable de al menos un 30\% de los delitos cometidos en el país (Blanco y Hein, 2010, Berríos, 2011). 
El sistema actual de ejecución penal juvenil presenta un modelo diferenciado del sistema de adultos(as), a cargo del Servicio Nacional de Menores (Sename) se basa en un modelo de justicia (Sename, 2012) que regula la aplicación de medidas y condenas desde un enfoque en los derechos y garantías de los/ las usuarios(as) del servicio, reconociéndoles como sujetos de derechos inalienables y en una etapa particular de desarrollo vital. Posee además un catálogo para la imposición de medidas y condenas que facilitaría la aplicación de sanciones acorde a cada joven, privilegiando sanciones en medio libre y se aplica a jóvenes entre 14 y 18 años, sin que por ello exista un límite etario dentro del cumplimiento de sanción.

El diseño de la ley se encuentra permeado por un enfoque en educación y que se traduce en orientaciones técnicas centradas en la promoción de procesos de inserción educativa. Pese a ello se ha observado que la educación como vía a la reinserción social no es percibida por los/las jóvenes como una oportunidad para mejorar su condición de vida en tanto no les aseguraría poder luego desarrollar una carrera laboral (Sename, 2012).

Esta situación fue observada también de primera mano por Rafael Rodríguez Walker ${ }^{1}$, quien durante su trabajo como delegado en un organismo colaborador acreditado del Sename en el año 2010 se dio cuenta de que no existían ofertas laborales estables y de sueldo acorde a mercado para sus usuarios(as) e intuyó que en la medida en que ello no ocurriera sería muy difícil lograr reinsertar socialmente a esto(as) jóvenes.

La evolución de la población del Sename durante los últimos años entrega luces sobre esta problemática pues se ha observado un creciente envejecimiento de la población atendida, llegando durante el año 2013 a 15.546 jóvenes, es decir, un 45,3\% del total de jóvenes atendidos(as) en este período.

Esto podría deberse a que, en primer lugar, el cumplimiento de las sanciones en la mayoría de los casos se da de manera sucesiva, por lo que un(a) joven puede pasar varios años en el servicio por delitos cometidos en un lapso breve de tiempo, algo que ocurre en parte importante de los casos pues acorde a cifras entregadas por el Sename, un 29,54\% de los/las jóvenes reincide antes de cumplirse un año de su ingreso a medidas en el medio libre o egreso de medidas privativas de libertad (Centro de Estudios en Seguridad Ciudadana [CESC], 2012).
Y, en segundo lugar, se ha observado que existen jóvenes que tras cumplir una condena en el sistema penal adulto deben retornar al Sename a cumplir sanciones que recibieron siendo menores de edad, sin que necesariamente sus planes individuales de intervención reflejen esta condición en tanto tienden a diseñarse de manera estandarizada (Comisión Constitución, Legislación, Justicia y Reglamento del Senado Chileno, 2013).

Ambas situaciones podrían estar detrás del hecho de que al día de hoy exista un número importante de jóvenes mayores de edad para quienes muchas veces los planes de intervención propuestos, basados en educación y familia, podrían no responder a sus necesidades, pudiendo beneficiarse del desarrollo de una carrera laboral. Con jóvenes como estos(as), que veían el trabajo como alternativa al delito, comenzó a trabajar en el año 2010 Fundación Proyecto B.

Inicialmente ideado como un voluntariado enfocado el emprendimiento laboral se centraba en entregar capacitación y recursos a jóvenes en cumplimiento de sanción bajo la LRPA, interesados(as) en desarrollar proyectos comerciales pertenecientes a distintas comunas de la Región Metropolitana.

Si bien en aquel entonces existían instituciones de larga trayectoria en el trabajo con personas condenadas, como Fundación Paternitas, la Corporación de Rehabilitación Social (Coresol) o el Instituto de Formación y Capacitación Popular (Infocap), sus programas no se centraban en entregar oportunidades a jóvenes del sistema de justicia juvenil, centrándose en la capacitación, sin ofrecer servicios de apoyo a la empleabilidad o intermediación laboral.

Durante el primer periodo de ejecución del programa 22 jóvenes ingresaron a distintas capacitaciones con el fin de generar sus propios emprendimientos, sin embargo se buscaba llegar a más jóvenes, ofrecerles nuevas oportunidades, y así se comenzó a desarrollar un programa basado en la colocación que incluía intervenciones psicoeducativas, capacitación en la empresa, acompañamiento a la familia y supervisión de el/la joven.

El pilotaje durante el año 2011 de ambos programas, al alero del Sename, llevó a establecer formalmente Proyecto B como una organización sin fines de lucro, en adelante Fundación Proyecto B. Esto coincidía con un aumento en el interés público por la reinserción social juvenil a través del desarro-

1 Psicólogo y MBA de la Pontificia Universidad Católica. Director ejecutivo y miembro fundador de Fundación Proyecto B. Se desempeña durante el año 2011 como delegado de Programa de Libertad Asistida Especial en Corporación PROMESI. 
llo laboral, impulsado en parte por el Ministerio de Justicia que incluía dentro de sus " 11 medidas para la reinserción juvenil” el eje de capacitación laboral. Acorde a resultados entregados por Sename, en ese año 2011 un 61,34\% de los/las jóvenes privados(as) de libertad (CRC y CSC) y en programas del medio libre (PLA-PLE) que cumplían con los requisitos mínimos para capacitación efectivamente accedieron a formación laboral (Sename, 2012).

Esta oferta programática consistía principalmente en cursos ofrecidos por el Servicio Nacional de Empleo y Capacitación (SENCE), talleres de empleabilidad y de alfabetización digital, además de becas individuales de formación para el trabajo y Programa Aprendices (Mertz, 2013).

Actualmente no solo existe una brecha importante de jóvenes, del medio libre especialmente, que no acceden a iniciativas de formación laboral durante ese periodo; ninguno de los programas de capacitación laboral ofrecidos por el servicio cuenta con evaluación de resultados, por lo que no puede conocerse en qué grado estas capacitaciones tuvieron una salida laboral sustentable, algo que se repite en otras organizaciones y que ha generado un vacío en cuanto al conocimiento disponible en el país respecto a experiencias de este tipo (Mertz, 2013).

Es en parte por ello que Fundación Proyecto B incluyó dentro de sus objetivos estratégicos poder aportar a generar evidencia empírica dentro del tema, generando durante el año 2012 su propia área de estudios, que estaría en adelante encargada de sistematizar, evaluar y mejorar el diseño del programa.

Tras una sistematización inicial del programa se comenzó a trabajar en la manualización de los procesos de la organización, con miras a la posibilidad futura de replicarlo en otras instituciones.

Para ello, en el año 2013 se evaluó la gestión del equipo ejecutor cumpliendo a cabalidad con los indicadores propuestos para ese periodo. Es en este año que el programa sufre las modificaciones más profundas en tanto se regulariza y ordena el tipo de intervenciones que realiza, se crea el Taller de Apoyo a la Empleabilidad, se rediseña el entrenamiento de actores y se modifican los criterios de ingreso y desvinculación.

A diciembre del año 2013 se había trabajado con 187 jóvenes en el programa de empleabilidad, logrando que más de la mitad de ellos cumpliese dos meses empleados y que casi un $40 \%$ se desempeñase 12 meses en la empresa.

Actualmente Fundación Proyecto B es una de las pocas organizaciones a nivel nacional que han traducido proyectos sociales a un manual operativo; contando con cinco documentos específicos que apoyan la ejecución y replicación de su programa de empleabilidad.

El presente documento tiene como objetivo presentar el modelo de intermediación laboral para jóvenes que han infringido la ley diseñado por Fundación Proyecto B, así como los resultados de su primera evaluación formal.

\section{Enfoque de intervención}

El programa de empleabilidad de Fundación Proyecto B para jóvenes que han infringido la ley se basa en un concepto integral de lo que implica la reinserción social, donde el trabajo es una de las aristas de cualquier intervención orientada a favorecer este proceso. En este sentido se considera que la reinserción social será todo

\section{"...proceso mediante el cual una persona que ha infringido la ley logra alcanzar una participación activa y prosocial en su entorno comunitario, la satisfacción de sus necesidades a través de medios lícitos y el ejercicio pleno de sus derechos sociales" (Morales, Welsch, Cárcamo, Aguilar y Sosa, 2013).}

Así, la reinserción social es vista como un proceso de desarrollo holístico, asociado a la disminución en la comisión de delitos y cuyo producto se determinaría en tanto la participación prosocial de la persona en sociedad y el ejercicio de derechos sociales tales como salud, educación y empleo.

\section{Adolescencia}

Si la transición desde una vida asociada al delito hacia el desistimiento delictual en general resulta compleja, para los/las jóvenes podría ser incluso más difícil, debido a factores de maduración, capacidad cognitiva y contexto psicosocial (Mears y Travis, 2004).

Se considera la adolescencia como aquella etapa del desarrollo biológico, psicológico, sexual y social que sigue a la niñez y que comienza con la pubertad cerca de los 13 años y que se comprende por fases, llegando la primera hasta los 14-15 años. La adolescencia media se extendería hasta los 19 años y la adolescencia tardía o juventud plena se extendería hasta los 24 años (Casas y González, 2005).

La curva etaria de la participación delictual muestra que la tasa de delitos según edad aumenta durante 
la adolescencia, alcanzando su peak durante la adolescencia tardía y la juventud plena. Hacia los 26 años la mayoría de los jóvenes habrá desistido del delito, por lo que si se busca tener un impacto importante sobre el desistimiento resulta importante centrar esfuerzos durante este peak (Miller, 2009).

Otro motivo para intervenir, específicamente desde un enfoque laboral, consiste en que todo contacto con el sistema de justicia, ya sea un arresto, causa o condena (en medio libre o cerrado), afecta negativamente la empleabilidad futura de el/la sujeto, generando distintas dificultades de tipo acumulativo que van consecutivamente afectando el proceso de desistimiento (Nagin \& Waldfogel, 1995; Waldfogel, 1994).

Como contraparte a estas barreras y con el afán de determinar específicamente qué elementos considerar a la hora de crear un programa para la reinserción social juvenil se ha indagado respecto a un enfoque para la intervención social basada en la investigación teórica que se describe a continuación.

\section{Enfoque de práctica basada en evidencia}

En el proceso de sistematizar la experiencia, y especialmente durante la reformulación de distintos elementos del Programa de Intermediación Laboral, se consideraron lineamientos clave aportados desde el enfoque basado en evidencia, respecto a aquellas características programáticas que serían más exitosas a la hora de trabajar con jóvenes que han infringido la ley.

Existen diversos cuestionamientos respecto a efectividad de los programas de reinserción social juvenil, sin embargo, acorde a Tennyson (2009) existirían ciertos factores que contribuirían a su éxito y que se relacionan estrechamente con los principios de efectividad basada en evidencia de la reinserción social con población adulta.

A continuación se presentan factores de éxitos descritos en la literatura internacional, distinguiendo en cada caso cuando estos no han sido posibles de incorporar al presente modelo de intermediación. Según la literatura combinada de Lipsey y Cullen (2007), Mackenzie (2006) y Sherman, Farrington, Welsh y Mackenzie (2002) los principios de efectividad, basados en evidencia, para programas juveniles de reinserción social son:

a. Identificación de necesidades criminógenas y evaluación del nivel de riesgo: Las intervenciones más intensas deberán ser dirigidas a usuarios/ as de mayor riesgo, atendiendo siempre a sus necesidades criminógenas particulares. Las ne- cesidades criminógenas corresponden a aquellos elementos en la vida de el/la sujeto que son susceptibles de modificación (dinámicas). Esto no ha sido posible incluirlo dentro del modelo de Fundación Proyecto B en tanto el desarrollo teórico nacional en torno a la evaluación de nivel de riesgo, disponible para la organización, no ha permitido acceder a un método de evaluación adaptado y validado para población nacional.

b. Adecuación del programa: Cada programa debe ser implementado teniendo en cuenta las características personales de el/la usuario(a), sin embargo, la etapa del desarrollo en que se encuentran los adolescentes está marcada por un constante cambio y representa un desafío a la hora de considerar distintos elementos como, por ejemplo, estilos de aprendizaje. Teniendo esto en cuenta, se ha considerado un diseño del programa que brinde la mayor flexibilidad posible, en términos de los contenidos a abordar y servicios a ofrecer, cuidando que estos sean adaptables a las características de cada joven.

c. Base teórica del programa: Aquellos programas desarrollados desde una teoría basada en evidencia empírica han demostrado mejores resultados en reducción de reincidencia que aquellos basados desde percepciones sociales de los/las ejecutores(as). En los casos donde el proceso ha sido inverso y los programas han sido diseñados desde la práctica, los procesos de manualización, sistematización y evaluación de la experiencia realizada resultan recomendables (Tennyson, 2009). Si bien desde sus inicios el programa diseñado por Fundación Proyecto B se basó en la experiencia particular de quienes lo crearon, la sucesiva sistematización y manualización del proceso ha permitido sustentar desde la teoría la práctica que se realizaba hasta la fecha y modificar aquella que no se encontraba respaldada por investigaciones en el área.

d. Dosificación del programa: Hace referencia a la calidad y cantidad del contacto que tienen quienes ejecutan un programa con los usuarios del programa. En el caso de los jóvenes resulta importante que la relación con los/las profesionales del programa sea estrecha y el contacto estable, aunque en jóvenes de bajo riesgo de reincidencia se da el efecto opuesto y un contacto excesivo puede resultar negativo. Asimismo, si los profesionales con los/las que interactúan los/las jóvenes se muestran poco motivados(as) y desinteresados(as) en su trabajo, los/las jóve- 
nes tenderán a imitar este comportamiento y a retirarse o disminuir su participación en la intervención (Stephenson, Giller y Brown, 2007).

e. Integridad del programa: Corresponde a la fidelidad de quienes ejecutan el programa respecto al diseño original de este. Existen distintas estrategias implementadas dentro del programa para ello, la primera consiste en establecer la supervisión y capacitación continua de todos(as) los/las ejecutores; algo que en el presente diseño se ha ubicado como responsabilidad del área de coordinación y de la dirección ejecutiva. La segunda, mencionada previamente, se aborda en la manualización del programa.

f. Modalidad del programa: Los programas más efectivos son aquellos que incluyen intervención cognitiva; entrenamiento en habilidades interpersonales, consejería individual y programas de entrenamiento conductual. Otro factor relevante es la participación voluntaria de los/las jóvenes y que influiría de manera importante en el éxito de la intervención (Tennyson, 2009).

Además de estos principios generales, existen una serie de descubrimientos respecto a la reinserción social a través del trabajo en particular que ciertamente aportan al diseño del programa:

\section{Características de programas no exitosos}

Lipsey, Wilson y Cothern (2000) observaron los resultados obtenidos por distintos programas de reinserción social juvenil y recopilaron algunos elementos que contribuirían al fracaso específico de programas de reinserción social a través del empleo para jóvenes que han infringido la ley y que concuerdan con aquellos elementos en la población latinoamericana juvenil general (Oficina Internacional del Trabajo [OIT], 2012):

a. No consideran las características del mercado laboral local. Así muchas veces los/las jóvenes son, por ejemplo, capacitados(as) en oficios de baja empleabilidad, sin que puedan luego acceder a emplearse debido a la escasa demanda que poseen. También puede ocurrir que sean capacitados(as) en oficios que requieren perfiles laborales distintos al suyo. La gestión de redes debe mantener siempre información actualizada respecto al mercado laboral nacional y a temas de empleabilidad.

b. No están en relación con las políticas públicas inclusivas existentes en el país por lo que sus usuarios(as) no pueden acceder a estos beneficios. c. Se enfocan solo en lo laboral dejando de lado otros servicios que requiere cada joven. Para ello el programa contempla la coordinación con el/ la delegado(a) de sanción de cada joven, quien estará a cargo de asegurar que el/la joven acceda a servicios que apoyen su inserción laboral.

\section{Características de programas exitosos}

Walker en un estudio publicado el año 1997 plantea algunos elementos a considerar, que elevarían la probabilidad de éxito de programas laborales:

a. Rápida colocación tras ingreso al programa o tras el fin de una capacitación. Periodos largos sin empleo tenderían a disminuir la motivación de los/las jóvenes provocando muchas veces incluso el abandono del programa.

b. Participación de adultos(as) significativos(as) que guíen y apoyen el proceso de cada joven, tanto desde el núcleo familiar como en la empresa.

c. Una buena relación con el/la empleador(a) aportaría al éxito del programa. El programa diseñado por Fundación Proyecto B considera el entrenamiento y la coordinación constante con distintos(as) trabajadores(as) de la empresa contratante de manera de facilitar una relación adecuada con los/las jóvenes insertos(as).

Así, se han intentado integrar los elementos de práctica efectiva al diseño completo del programa. A continuación se presenta el marco conceptual que se ha construido teniendo en cuenta aquellos modelos teóricos que darían una explicación más cercana a la práctica del programa respecto al proceso de reinserción social.

\section{Enfoque ecológico y modelo de desarrollo social}

Dentro de los distintos enfoques de análisis de la conducta humana que existen en la literatura internacional se ha optado por la ecología del desarrollo humano desarrollada por Bronfenbrenner (1987) y que conceptualiza el comportamiento humano como resultado de una relación dinámica entre la persona y su contexto.

Este enfoque proporciona un buen marco teórico a la hora de comprender la complejidad de las causas ambientales que explican la conducta humana en tanto permite dar cuenta de la relación de cada persona respecto a los distintos sistemas en que se encuentra inserto(a). En la ilustración que se presenta a continuación se han graficado estos sistemas y que corresponden a: 
- Microsistema: Contexto inmediato en que se encuentra una persona (familia, escuela o trabajo).

- Mesosistema: Relaciones establecidas entre los diversos microsistemas. Por ejemplo, la gestión de entrega de servicios, realizada por el programa, busca actuar a nivel de mesosistema

- Exosistema: Estructuras sociales que influyen sobre los entornos específicos que contienen a el/la sujeto. Por ejemplo sistemas de representaciones sociales relativas a la pobreza.

- Macrosistema: Esquemas y valores culturales del cual los niveles anteriores son manifestaciones concretas. Por ejemplo, la estructura patriarcal o la macroeconomía neoliberal.

Reformulaciones posteriores de la teoría inicial han definido a aquellos eventos y características en el entorno que influirían en el desarrollo del sujeto, como variables más o menos susceptibles de modificación (Valdenegro, 2005).

\section{Modelo desarrollo social y enfoque de riesgo}

Este modelo (en adelante MDS), al igual que el enfoque ecológico presentado previamente, se configura como una teoría general del comportamiento humano pero que pone un énfasis en explicar el origen de la conducta contraria a la norma social (antisocial) y su contraparte, el comportamiento acorde a la norma (prosocial) (Catalano y Hawkins, 1996; Catalano y Kosterman, Hawkins, Newcomb, y Abbott, 1996; Lonczak, Huang, Catalano, Hawkins, Hill, Abbott, Ryan y Kosterman, 2001).

Para ello el MDS integra elementos de tres de las principales teorías que han intentado describir la etiología del comportamiento antisocial; la teoría del control social (Hirschi, 1969), teoría del aprendizaje social (Bandura, 1987) y la teoría de asociación diferencial (Sutherland, 1970).

De acuerdo con el MDS, los individuos aprenden patrones de conducta prosocial o antisocial en distintos grupos socializadores, como la familia, los pares, la escuela, etc. Cuando un(a) niño(a) tiene acceso a oportunidades de participación en estas instancias, desarrolla habilidades para participar exitosamente en esos espacios y su participación es reconocida, genera un vínculo con el grupo socializador, aumentándose la probabilidad de que adquiera las creencias y valores pertenecientes a ese grupo, y estas creencias y valores moldearán su conducta (Choi, Harachi, Rogers \& Catalano, 2005).

Así, existirían desde el MDS tres elementos que irán configurando el desarrollo de la persona hacia una identidad y conductas prosociales o hacia conductas antisociales:

- Oportunidades de participación.

- Desarrollo de habilidades para la participación.

- Reconocimiento y valoración de la participación.

Dependiendo de cómo se den estas tres instancias cada niño(a) o adolescente irá integrando las normas sociales de los grupos donde participe, y ello irá aumentando su probabilidad de desarrollarse de prosocial o antisocial.

Algunos estudios desarrollados en cuando a qué elementos del entorno llevarían a los/las jóvenes por uno u otro "camino" se han enmarcado dentro de la llamada perspectiva de riesgo, cuyo eje central son los factores de riesgo y protección, y que se presenta a continuación:

\section{Factores de riesgo}

Corresponden a características individuales y del entorno (inmediato o distante), que aumentan la probabilidad de que las personas externalicen comportamientos de riesgo como la deserción escolar, el abuso de sustancias o el comportamiento delictivo, entre otros (Hein, 2004).

Dentro de los factores de riesgo que se relacionan con la aparición de conductas de riesgo asociadas al delito se encuentran factores individuales, familiares, factores ligados al grupo de pares, factores escolares sociales o comunitarios y socioeconómicos y culturales.

La presencia factores de riesgo aumentarían, como se mencionó previamente, la probabilidad de que una persona presente conductas de riesgo y que Hein (2004), tras una extensa revisión, clasificó en cuatro grupos principales:

a. Uso y abuso de alcohol y drogas.

b. Relaciones sexuales no protegidas.

c. Bajo rendimiento, fracaso y deserción escolar.

d. Infracción penal y violencia.

La probabilidad de que aparezcan estas conductas llamadas de riesgo se ve mediada por elementos ya sea individuales o contextuales llamados de protección y que se describen a continuación.

\section{Factores de protección}

Los factores de protección o factores protectores no implican de ninguna manera la ausencia de factores de riesgo, sino más bien la presencia de elementos que modulen el efecto que tienen estos sobre la pro- 
babilidad de que aparezca una conducta de riesgo (Hein, 2004).

En ese sentido, los factores de protección disminuyen la probabilidad de que la persona genere comportamientos no deseados, pese a la presencia de factores de riesgo.

El programa de Fundación Proyecto B se centra en los factores de protección, en tanto no busca necesariamente disminuir la presencia de factores de riesgo, sino que introducir factores de protección a través de la colocación, capacitación o el Taller de Apoyo a la Empleabilidad, que disminuyan la probabilidad de que un(a) joven se involucre en actividades delictuales.

Esto se realiza tanto a través de la intervención en factores de protección individuales como en el fortalecimiento del vínculo de el/la joven con la sociedad a través de su vinculación con el lugar de trabajo y con profesionales del programa como a través de factores contextuales como la relación con pares de creencias prosociales.

A continuación se presentan aquellas cualidades que se relacionan fuertemente con las nociones del Modelo de Desarrollo Social presentado previamente y que disminuirían la probabilidad de aparición de conductas problemáticas (Hein, 2004):

a. Presencia de adultos(as) significativos(as) de creencias prosociales.

b. Altas expectativas por parte de personas en el entorno.

c. Oportunidades de participación significativa.

A estos tres elementos, considerados en la creación del programa de intermediación laboral presentado en este documento, se suman factores a nivel individual que disminuirían la probabilidad de participación en conductas de riesgo y que se han integrado específicamente dentro del Taller de Apoyo a la Empleabilidad. (Bernard, 1995):

a. Habilidades interpersonales: Poseer las competencias necesarias para provocar respuestas positivas en los demás, tales como flexibilidad, empatía, comunicación efectiva y sentido del humor.

b. Habilidades de solución de problemas: Ser capaz de planificar, poseer pensamiento crítico, creativo y reflexivo. También se considera como una habilidad importante el conocer a personas a quienes poder acudir para ayuda en caso de tener problemas.

c. Conciencia crítica: Ser capaz de reflexionar (darse cuenta) de las circunstancias negativas en las que se vive, además de poder crear estrategias para superarlas.

d. Autonomía: Poseer una noción de la propia identidad, habilidad para actuar en forma independiente, ser capaz de autocontrolarse y mantener un sentido de autoeficacia

e. Sentido de propósito: Tener aspiraciones, motivación de logro, persistencia, esperanza, optimismo y capacidad de dirigir el comportamiento al cumplimiento de metas.

Tanto el modelo de desarrollo social como la perspectiva de factores de riesgo y el concepto de factores de protección comparten como elemento común la noción de que distintos elementos individuales y contextuales, según cómo interactúen, aumentarán o disminuirán la probabilidad de que un(a) joven presente conductas problemáticas.

Se pone énfasis en el concepto "conductas problemáticas" en tanto el modelo ecológico, el modelo de desarrollo social y la perspectiva en riesgo pueden aplicarse a distintos comportamientos problemáticos, donde la infracción penal representa solo uno de los grupos mayoritarios de conductas estudiadas.

\section{Reinserción social con enfoque laboral}

La participación, durante la adolescencia temprana, en actividades delictuales, dificulta el desarrollo educacional y laboral en las etapas posteriores. Ello debido a que las dificultades sociales tienden a ser acumulativas y lo que comienza como una barrera para el acceso a educación de calidad, por ejemplo, deriva en complicaciones para el posterior acceso a puestos de trabajo estables y bien remunerados (Tanner, Davies y O'grady, 1999, Uggen y Wakefield, 2003).

Esto genera una reacción en cadena que podría mantener a el/la joven desempleado(a) durante un largo plazo, dificultando su desistimiento del delito, especialmente si es que el proceso de transición entre adolescencia y adultez se da intramuros (Uggen y Wakefield, 2003).

Es por ello que una de las problemáticas en reinserción, y que ha tomado relevancia en el país durante los últimos 10 años, consiste en la reinserción social de enfoque laboral y que incluye tanto la intermediación laboral, como la capacitación, colocación, apoyo a la empleabilidad y acompañamiento, entre otros.

\section{Empleabilidad e intermediación laboral}

La empleabilidad puede ser definida como la capacidad que tendría una persona de obtener un 
empleo que satisfaga sus necesidades laborales. El apoyo a la empleabilidad consistirá, por lo tanto, en aumentar la posibilidad de que una persona, en el mercado laboral, pueda acceder a un empleo con las características que desea. Así, el apoyo a la empleabilidad se relacionaría con promover que una persona aumente sus posibilidades de optar y desempeñarse laboralmente (Martín y Spinosa, 2005)

La intermediación laboral por su parte puede definirse como una estrategia para el aumento de la empleabilidad, basada en la gestión o entrega de servicios de capacitación en oficios técnicos y habilidades blandas laborales, la gestión de colocación laboral y la coordinación entre actores relevantes.

Como se mencionó anteriormente, el enfoque laboral basado en intermediación y empleabilidad ha tomado relevancia durante los últimos años como herramienta para la reinserción social de personas condenadas y si bien los estudios meta-analíticos indicarían que este tipo de programas tiene una mayor efectividad en usuarios(as) mayores de 26 años, existen estudios que sugieren que también podría ser útil para promover el desistimiento en jóvenes cuando se combina con actividades de formación y la empresa se configura como un lugar donde compartir con pares de creencias prosociales (Tennyson, 2009).

Así mismo, y respecto al rol de los programas de intervención, el mantener un apoyo psicosocial por seis meses hasta varios años después de la colocación efectiva contribuiría a un mayor tiempo de mantención en el trabajo y aumento de los ingresos promedio (Mulvey, 2011).

La oportunidad de desempeñarse junto a pares de creencias prosociales, así como la presencia de un(a) asesor(a) laboral con quien establecer una relación de trabajo aportarían al desistimiento en tanto el vínculo con personas de creencias prosociales y con la institución social del trabajo se constituyen como una fuente potente de control social informal sobre la conducta delictual (Hirschi, 1969; Sampson \& Laub, 1993).

Este vínculo con el trabajo además motivaría el desistimiento en tanto entrega una "oportunidad que cuidar" a la persona, especialmente si es un empleo estable, de ingresos acorde al mercado, pues vendría a representar un punto de cambio para los individuos, entregándoles un mayor capital social y mayor inversión en relaciones institucionales convencionales (Hagan \& McCarthy, 1997; Sampson \& Laub, 1993).

El solo hecho de completar un programa de intermediación laboral ha demostrado tener efecto en la disminución de reincidencia y aumento de la empleabilidad (Wilson, Gallagher y Mackenzie, 2000), especialmente si incluye una colocación en un empleo de calidad. En un estudio en profundidad realizado por Uggen (2003) se observó que existiría evidencia suficiente para afirmar que el acceso a empleos de calidad; de remuneración mayor al mínimo, estables y que satisfagan las necesidades e intereses de los/las usuarios(as), tendría un efecto positivo en la reducción de las probabilidades de los/las usuarios(as) de reincidir y aumentaría su empleabilidad.

De acuerdo con la literatura internacional, la colocación, el apoyo a la empleabilidad y la capacitación laboral contribuirían de distinta manera a la reinserción social de las personas en conflicto con el sistema penal donde la colocación tendría el menor efecto, aumentando cuando se incluye acompañamiento y especialmente cuando se incluye capacitación (Henry y Jacobs, 2007; Mackenzie, 2006; Seiter, 2004; Villagra, 2007).

Dicho efecto podría deberse a que el tener un empleo estable, de remuneración acorde al cargo y con condiciones laborales adecuadas no solo entrega a la persona una fuente legítima de dinero y reduce su tiempo disponible para participar en actividades delictuales sino que le permite establecer nuevos vínculos interpersonales y sociales que sirvan como forma de control social y minimicen o eliminen la asociación con pares de creencias y conductas antisociales (Kazemian y Maruna, 2009; Laub \& Sampson, 2001).

Este último punto es especialmente importante en el caso de población juvenil, para quienes la influencia de pares sería crucial y a quienes beneficiaría especialmente el tener colegas prosociales que sirvan como modelos a seguir (Hein, 2004; Maruna, 2001). Otras variables como cumplir con 6 meses de acompañamiento o terminar un programa de empleabilidad por sí solos tendrían un efecto en la disminución de reincidencia o aumento de empleabilidad (Monahan, Steinberg y Cauffman, 2012; Uggen y Wakefield, 2003).

\section{Intermediación laboral a través del manejo de casos y tutoría}

Encontrar un empleo requiere no solo de las competencias particulares de un individuo, sino que de una red de relaciones de cada individuo y su comunidad que le conecten con un empleo, le apoyen y mantengan ahí. De hecho se estima que el 80\% de los empleos no son nunca anunciados públicamente. Es por ello 
que el capital social de una persona resulta clave para mejorar su empleabilidad (Brown y Ross, 2010).

La metodología de manejo de casos ve a el/la asesor(a) laboral como la primera sección de esta red de relaciones. Esta metodología es relativamente nueva dentro de la reinserción social y emerge debido a que se comenzó a considerar insuficiente el supervisar o acompañar a el/la usuario(a), sino que se requeriría un conjunto de acciones que promuevan conductas prosociales, generen confianza, motiven el proceso de cambio y potencien la formación de la persona. Así, la persona a cargo del caso cumpliría un rol no solo de control social y gestión de servicios, sino que además debiese generar un vínculo para la intervención social (Droppelmann, 2010).

\section{Diseño programático}

El programa posee cinco componentes principales que se ejecutan según las particularidades de cada joven, su proceso dentro de él y la disponibilidad de cupos de empleo y capacitación, los cuales pueden observarse en el esquema general presentado en la figura 1. Puede, por ejemplo, ingresar el/la joven a capacitación y luego ser colocado(a) directamente o continuar con su Taller de Apoyo a la Empleabilidad en caso de demorarse la apertura del cupo de empleo. En este sentido, cada componente corresponde a un servicio o actividad a realizarse y que se puede flexibilizar acorde al proceso particular de cada joven ingresado(a) al programa.

Componente prelaboral: Previo a que un(a) joven ingrese al programa deben realizarse una serie de actividades que buscan, en primer lugar, establecer convenios de trabajo conjunto con las instituciones y empresas que permitirán llevar a cabo el programa. Luego se realiza un entrenamiento para todas las personas que trabajarán directamente con cada joven, ya sea en organismos de capacitación técnica, en la empresa o quienes realizan su seguimiento de sanción. El objetivo de este entrenamiento es alinearse respecto a los objetivos del programa y entregar recomendaciones para potenciar el proceso de inserción de cada joven.

En el caso de la empresa se requiere, además de la coordinación del programa, se designa un(a) jefe(a) director(a) de cada joven, con quien se realizará la evaluación de competencias blandas, y otro(a) trabajador(a) que esté a cargo de la tutoría de el/la joven, acompañando de manera informal su inserción laboral.

Finalmente se realiza la selección de los/las jóvenes que ingresarán al programa a partir de un modelo particular, diseñado por Fundación Proyecto B, que combina la práctica y saber institucional con un diseño basado en competencias que permite seleccionar a los/las candidatos(as) sobre la base del cargo donde se desempeñarán y disminuir al mínimos los criterios personales no sistematizados que guíen la selección.

Esta selección evalúa factores psicosociales que puedan dificultar o facilitar la inserción laboral, competencias blandas y/o técnicas requeridas para el cargo a postular y criterios programáticos de ingreso. Estos últimos corresponden a tener más de 18 años, no cumplir con criterios de dependencia de drogas y contar con un estado de salud compatible con el cargo.

Componente capacitación: Cada vez que resulta posible gestionar cupos de capacitación técnica para los/ las jóvenes del programa, se busca ejecutar cursos de alta empleabilidad, es decir, en oficios con alta demanda en el mercado laboral. Así mismo, se ase-

FIGURA 1

ESQUEMA GENERAL DE LOS COMPONENTES DEL PROGRAMA DE INTERMEDIACIÓN LABORAL DE FUNDACIÓN PROYECTO B PARA JÓVENES QUE HAN INFRINGIDO LA LEY. ELABORACIÓN PROPIA.

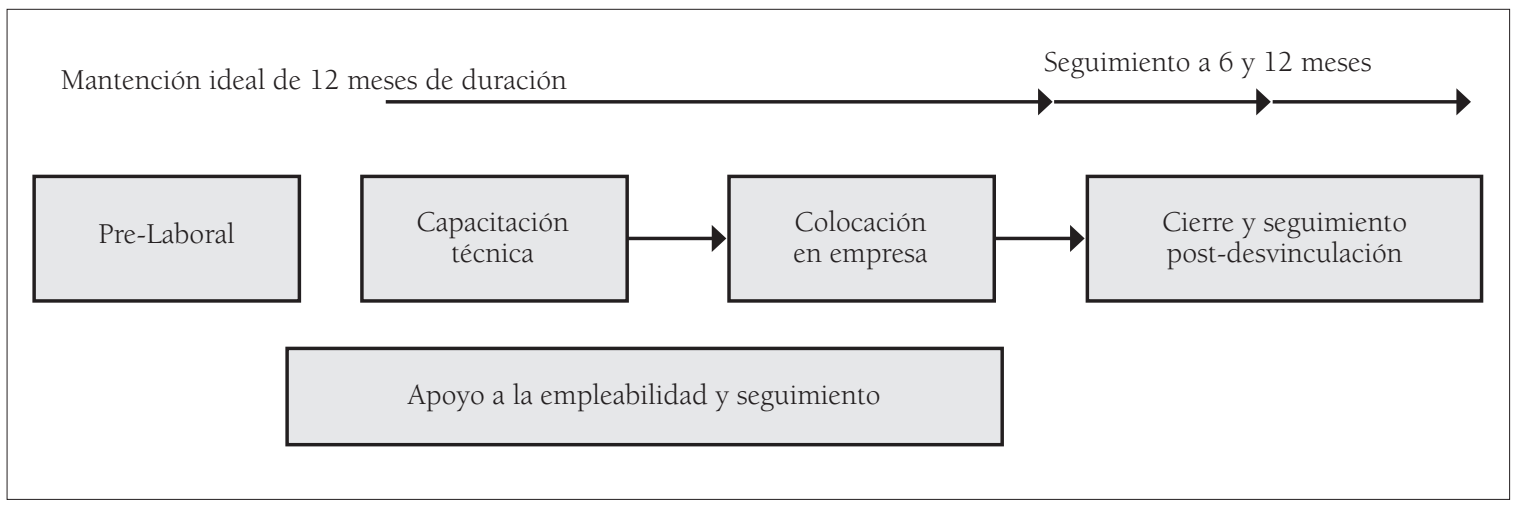


guran cupos de colocación previo al ingreso de los/ las jóvenes, promoviendo y motivando su participación con una oferta laboral real de contratación una vez terminado el curso.

Durante la capacitación se monitorea desde fuera de la sala de clases el desarrollo de cada curso, prestando asistencia a los/las profesores(as) cuando fuese necesario. Ambos factores (la colocación asegurada y el monitoreo en clase) se consideran cruciales para el éxito que han tenido las capacitaciones llevadas a cabo a la fecha, las cuales han sido completadas por un 90\% de los/las jóvenes ingresados(as).

Componente colocación: Insertar laboralmente a jóvenes que han infringido la ley requiere de empresas que estén dispuestas no solo a abrir cupos laborales en puestos de trabajo formales, estables y de remuneración acorde al cargo, sino que además estén comprometidas con la reinserción social de los/las jóvenes y comprendan las particularidades del trabajo que se busca realizar.

En este sentido se promueve que los puestos laborales en los que sean colocados constituyan un espacio de aprendizaje ligado a la práctica, donde sus superiores puedan fomentar su formación en habilidades específicas al puesto de trabajo y donde se facilite su acceso a capacitaciones técnicas certificadas.

Asimismo, y si bien cada empresa puede mantener su propio sistema de contratación, bonificación y desarrollo de carrera para potenciar el resultado exitoso del programa, a partir de la experiencia de Fundación Proyecto B, se sugiere diseñar un modelo de micrologros a lo largo de la inserción que motiven la continuidad y mejora de cada joven en su puesto de trabajo.

También se espera que el/la empleador(a) le entregue las facilidades para que cada joven asista a su centro de cumplimiento de sanción, sin que ello vaya en perjuicio de su sueldo mensual.

Componente apoyo a la empleabilidad y seguimiento: $\mathrm{Al}$ ingresar un(a) joven al programa, se comienza a ejecutar el componente de apoyo a la empleabilidad y seguimiento que busca brindar a los/las participantes del programa apoyo psicosocial y formación en competencias blandas para la empleabilidad que promuevan su mantención en el lugar de trabajo, así como un monitoreo continuo que permita supervisar y potenciar, a través de la coordinación con otros actores relevantes, su desempeño en la capacitación o empresa. Este componente posee por tanto dos subcomponentes.

Apoyo a la empleabilidad: El Taller de Apoyo a la Empleabilidad tiene como objetivo no solo acompañar el proceso de inserción laboral de el/la joven a través del establecimiento de un vínculo estable con su asesor(a) laboral, sino que entregarle herramientas que faciliten su inserción exitosa dentro de su empleo y que contribuyan al mantenimiento de conductas prosociales adquiridas durante su participación en el programa, además de aumentar su empleabilidad futura.

Este taller ha sido confeccionado tras la revisión de materiales de empleabilidad como el Programa Competencias Laborales de Fundación Chile, el toolkit de apoyo a la empleabilidad del proyecto KC4All y materiales relacionados con la reinserción social de jóvenes que han infringido la ley, como el Programa Detour (UMATERR Project, 2013), el Programa Thinking 4 a Change (National Institute of Corrections [NIC], 2011), el Programa Central de Tratamiento Educativo y Terapéutico para Menores Infractores de la Agencia de la Comunidad de Madrid para la Reeducación y Reinserción del Menor Infractor (Graña y Rodriguez, 2012), todos basados en modelos cognitivos. Cada temática se ha complementado además con bibliografía específica en el tema y con la experiencia del equipo en terreno de Fundación Proyecto B.

Las 24 sesiones del taller, distribuidas en 2 sesiones mensuales durante 12 meses desde el ingreso de el/ la joven al programa, se clasifican en tres módulos:

- Módulo de apresto laboral: Tiene como objetivo preparar para a el/la joven para su ingreso a la empresa o en la búsqueda de trabajo.

- Módulo de competencias blandas: Incluye temáticas relacionadas con la introducción al mundo laboral, el desarrollo de habilidades interpersonales básicas y complejas, la identidad asociada al trabajo, entre otras. Idealmente debiese desarrollarse tanto en la empresa o capacitación de el/la joven como en su hogar, maximizando las cualidades positivas de cada entorno.

- Módulo de cierre: Previo a la salida de un/a joven del programa se revisan en detalle los motivos de desvinculación, destacando aprendizajes a partir de la experiencia y generando estrategias de mantención de los aprendizajes obtenidos.

Se optó por el trabajo a través de un taller en tanto permite no solo que los/las usuarios(as) accedan al conocimiento que se considera necesario comprendan sino que además brinda un espacio para ponerlo en práctica a través de distintas actividades que promueven la reflexión en torno a lo aprendido. Las temáticas que abordará el TAE, así como las actividades diseñadas, se encuentran en el Manual de Empleabilidad para Asesores(as) Laborales. 
Cada asesor(a) laboral deberá por lo tanto revisar en detalle este manual con el fin de manejar no solo los conceptos a abordar, sino que además conocer las distintas actividades y aplicarlas a lo largo del acompañamiento del joven.

En las ocasiones en que distintos eventos en la vida de el/la joven requieran que se realice una visita no planificada (extraordinaria) deberá su asesor(a) laboral aprovechar de generar una instancia de aprendizaje, integrando contenidos del taller relevantes a la visita. Siempre debiese priorizarse la realización de una actividad de formación para la empleabilidad.

Seguimiento: Con el fin de promover la inserción en un empleo o capacitación de cada joven y asistir en la resolución de cualquier dificultad que emerja durante este proceso, se realiza un seguimiento constante a todos(as) los/las jóvenes participantes del programa. Ello a través de actores clave en el proceso, como jefes(as) directos(as) o tutores(as) en la empresa, profesores(as) en el caso de cursos de capacitación y finalmente delegados(as) de sanción. La periodicidad de este contacto varía según el periodo que lleve inserto(a) cada joven; contacto semanal durante el primer mes, quincenal durante los siguientes cinco meses y mensual durante el último semestre de programa. Este seguimiento dura por tanto 12 meses y comienza una vez que el/la joven ingresa al programa.

\section{Componente cierre y seguimiento posdesvincu- lación}

Ya sea debido a que el/la joven ha completado 12 meses desde el ingreso al programa o debido a que ha incumplido con la normativa del programa, se deberá realizar un cierre del proceso de inserción, entregando retroalimentación respecto a la inserción de el/la joven a todos(as) los actores relevantes.

\section{Resultados}

A diciembre del año 2013 el programa había trabajado con 187 jóvenes en su programa de empleabilidad, logrando que más de la mitad de ellos cumpliese dos meses empleados y que casi un $40 \%$ cumpliese 12 meses en la empresa y se planteó el siguiente desafío: trasladar esta experiencia hacia otras instituciones del país.

Con este objetivo se realizó un proyecto en conjunto con el Servicio Nacional de Menores en el cual se sometió el diseño del programa a revisión extensa por parte del servicio, las empresas en convenio con Fundación Proyecto B, académicas nacionales expertas en el área de justicia y reinserción, así como con ejecutores(as) del programa.

Dentro del proyecto se observó la tasa de reincidencia de jóvenes participantes del programa. Para efectos de esta evaluación se consideró reincidencia como toda condena recibida tras la desvinculación del programa, estuviese el/la joven cumpliendo sanción o no.

Debido a falencias en el acceso a datos solo se realizó un análisis descriptivo de la reincidencia presentada por jóvenes que fueron colocados(as) por el programa durante el año 2012, realizando un seguimiento de 12 meses desde su salida, considerándose como reincidencia toda aquella nueva condena recibida por el/la joven después de su desvinculación del programa, contabilizándose por separado nuevas formalizaciones como dato respecto a nuevos contactos con el sistema penal.

Los datos fueron entregados por levantados por la dirección nacional el Sename a través de búsqueda en el sistema SIAG, incluye datos respecto a sanciones en el marco de la LRPA y al sistema penal adulto. La muestra completa comprendía 57 RUTS que se han detallado en el cuadro 1.

\section{DESCRIPCIÓN DE LA MUESTRA PARA MEDICIÓN DE TASA DE REINCIDENCIA}

\begin{tabular}{|l|l|}
\hline Edad al ingresar al programa & $\begin{array}{l}\text { En promedio tenían } 18 \text { años al ingresar, } 46 \text { jóvenes mayores de } 18 \text { años (80,7\%), 10 } \\
\text { jóvenes menores de } 18 \text { años }(17,5 \%), 1 \text { sin información de edad }(1,8 \%) .\end{array}$ \\
\hline Género con el que se identifica & Durante ese año ingresaron al programa 3 mujeres (5,2\%) y 54 hombres (94,8\%) \\
\hline $\begin{array}{l}\text { Delito por el cual cumplía } \\
\text { sanción al ingresar al programa }\end{array}$ & $\begin{array}{l}\text { Solo } 1 \text { joven }(1,8 \%) \text { ingresó por tráfico de drogas, el resto de los/las jóvenes ingresó por } \\
\text { delitos contra la propiedad }(98,2 \%) \text {. En el caso de los jóvenes que ingresaron por delitos } \\
\text { contra la propiedad la mayoría ingresó por robo con intimidación (32;57,1\%), le siguen } \\
\text { aquellos por robo con violencia (15;26,8\%), luego robo en lugar habitado (7; } 12,5 \%), \text { un } \\
\text { joven por hurto y un joven por robo en lugar no habitado (1,8\% respectivamente). }\end{array}$ \\
\hline Rubro en el que fue colocado(a) & $\begin{array}{l}\text { Los/las jóvenes fueron colocados(as) principalmente en empresas de la construcción (21, } \\
36,8 \%), \text { seguidos por rubros de industrias para la construcción (14, 24,6\%) y mecánica } \\
\text { automotriz (15, 26,3\%). Un menor número de jóvenes fue colocado(a) en área de servi- } \\
\text { cios }(7,12,3 \%)\end{array}$ \\
\hline
\end{tabular}

Elaboración propia. 
Al analizar los datos entregados por Sename, presentados en el cuadro 2 , se pudo observar que solo el $17,7 \%$ de los/las jóvenes desvinculados(as) del programa durante el año 2012 recibió una nueva condena dentro del año posterior a su salida. Si bien esta cifra no es comparable con la población total de Sename, o con otros programas en tanto no existen evaluaciones de programas similares, sí se considera promisoria en tanto la muestra está compuesta por jóvenes que pertenecen a perfiles delictuales que se ha observado tienen una mayor probabilidad de reincidir (acorde a estudios nacionales de Sename y Fundación Paz Ciudadana en la materia).

CUADRO 2

TASA DE REINCIDENCIA GENERAL

\begin{tabular}{|l|c|c|c|}
\hline & Nueva Ccausa & Nueva sanción & Total grupo \\
\cline { 1 - 3 } Reincidencia general & 29 & 10 & 57 \\
\cline { 1 - 2 } \%eincidencia general & $50,90 \%$ & $17,50 \%$ & 57 \\
\hline
\end{tabular}

Elaboración propia.

Si se observan los resultados clasificados por grupo etario en el cuadro 3 podrá notarse que, si bien la mayoría de jóvenes reincidentes son mayores de 18 años, en relación al total de cada grupo podrá observarse que el número de jóvenes mayores de 18 años que recibió una nueva condena es mucho menor.

CUADRO 3

TASA DE REINCIDENCIA SEGÚN GRUPO ETARIO

\begin{tabular}{|c|c|c|c|}
\hline \multirow{2}{*}{ Edad } & Nueva causa & Nueva sanción & Total por edad \\
\hline \multirow{2}{*}{$15-17$ años } & 8 & 4 & 10 \\
\cline { 2 - 3 } & $80,00 \%$ & $40 \%$ & 45 \\
\cline { 2 - 3 } 18 y más años & 20 & $11,1 \%$ & 2 \\
\cline { 2 - 3 } & $44,40 \%$ & 2 & 2 \\
\hline \multirow{2}{*}{ S/I } & 2 & $100 \%$ & 2 \\
\hline
\end{tabular}

Elaboración propia.

Por otra parte, al distinguir jóvenes según el tiempo de mantención dentro del programa, cómo se presentan en el cuadro 4 , puede observarse que la mayoría de los/las jóvenes reincidentes se había mantenido menos de dos meses en el programa (60\%), no obstante ello, respecto a cada grupo, el mayor porcentaje de reincidentes se da dentro del grupo que duró 6 a 9 meses (50\% reinciden en ese grupo).

Los menores porcentajes de reincidencia se observan en jóvenes que duran entre 2 y 6 meses y jóvenes que cumplieron 12 meses en el programa $(5,6 \%)$, se observan menos contactos con el sistema de justicia en el segundo grupo mencionado.

\section{CUADRO 4}

TASA DE REINCIDENCIA SEGÚN TIEMPO DE MANTENCIÓN EN LA EMPRESA

\begin{tabular}{|c|c|c|c|}
\hline Tiempo mantención & Nueva causa & Nueva sanción & Total grupo según mantención \\
\hline \multirow{2}{*}{$0-2 \mathrm{~m}$} & 9 & 6 & 17 \\
\cline { 2 - 4 } & $52,9 \%$ & $35,3 \%$ & 18 \\
\cline { 2 - 4 } & 13 & $5,6 \%$ & 4 \\
\hline \multirow{2}{*}{$2-6 \mathrm{~m}$} & $72,2 \%$ & 2 & 18 \\
\hline \multirow{2}{*}{$6-9 \mathrm{~m}$} & 3 & $50 \%$ & 18 \\
\hline
\end{tabular}


Al cruzar ambas clasificaciones se puede observar, según se presenta en el cuadro 5, que las mayores tasas de reincidencia se dan en el grupo menor de 18 años, específicamente en los grupos que duraron menos de 2 meses o de 6 a 9 meses. Debido al bajo número de casos estudiados no puede inferirse más allá de estos datos, sin embargo debe considerarse a la hora de aplicar el programa respecto a la inclu- sión de jóvenes menores de 18 años.

No sorprende que la menor proporción de reincidentes se encuentre en el grupo mayor de 18 años que duró 12 meses en el programa, pues se ha observado en la práctica que aquellos(as) jóvenes de mayor edad y que se mantienen más tiempo en él al egresar dan cuenta de mayores cambios a nivel emocional, cognitivo y familiar.

CUADRO 5

TASA DE REINCIDENCIA SEGÚN TRAMO ETARIO Y TIEMPO DE MANTENCIÓN EN LA EMPRESA

\begin{tabular}{|c|c|c|c|c|}
\hline Edad & Mantención & Nueva causa & Nueva sanción & Total grupo \\
\hline \multirow{8}{*}{ 15-17 años } & \multirow{2}{*}{$0-2 \mathrm{~m}$} & 3 & 2 & \multirow{2}{*}{3} \\
\hline & & $100 \%$ & $67 \%$ & \\
\hline & \multirow{2}{*}{$2-6 m$} & 3 & 0 & \multirow[b]{2}{*}{4} \\
\hline & & $75 \%$ & $0 \%$ & \\
\hline & \multirow{2}{*}{$6-9 m$} & 2 & 2 & \multirow{2}{*}{2} \\
\hline & & $100 \%$ & $100 \%$ & \\
\hline & \multirow{2}{*}{$12 \mathrm{~m}$} & 0 & 0 & \multirow{2}{*}{1} \\
\hline & & $0 \%$ & $0 \%$ & \\
\hline \multirow{8}{*}{18 y más años } & \multirow{2}{*}{$0-2 m$} & 5 & 3 & \multirow{2}{*}{12} \\
\hline & & $42 \%$ & $25 \%$ & \\
\hline & \multirow{2}{*}{$2-6 m$} & 10 & 1 & \multirow{2}{*}{14} \\
\hline & & $71 \%$ & $7 \%$ & \\
\hline & \multirow{2}{*}{$6-9 m$} & 1 & 0 & \multirow{2}{*}{2} \\
\hline & & $50 \%$ & $0 \%$ & \\
\hline & \multirow{2}{*}{$12 \mathrm{~m}$} & 4 & 1 & \multirow{2}{*}{17} \\
\hline & & $23,5 \%$ & $5,9 \%$ & \\
\hline \multirow{2}{*}{$\mathrm{S} / \mathrm{I}$} & & 2 & 2 & \multirow[b]{2}{*}{2} \\
\hline & & $100 \%$ & $100 \%$ & \\
\hline
\end{tabular}

Elaboración propia.

Respecto a la comparación según rubros de empresas en la que jóvenes fueron colocados(as), y que se presenta en el cuadro 6 , la mayor tasa de reincidencia se dio en el sector de la construcción (24\%), seguido por el sector de industrias para la construc- ción (21\%). Esto resulta especialmente relevante de considerar a la hora de realizar la apertura de cupo, así como las características particulares del tipo de empresa y cargos que cada rubro ofrece.

CUADRO 6

TASA DE REINCIDENCIA SEGÚN RUBRO DE COLOCACIÓN

\begin{tabular}{|l|c|c|c|c|c|}
\hline & Nueva causa & \% nueva causa & Nueva condena & \% nueva sanción & Total por rubro \\
\hline Construcción & 14 & $67 \%$ & 5 & $24 \%$ & 21 \\
\hline Industria & 5 & $36 \%$ & 3 & $21 \%$ & 14 \\
\hline Mecánica & 7 & $47 \%$ & 2 & $13 \%$ & 15 \\
\hline Servicios & 3 & $43 \%$ & 0 & $0 \%$ & 7 \\
\hline
\end{tabular}

Elaboración propia. 
Al observar los delitos por los que fueron sancionados(as) y que se han incluido en los cuadros 7 y 8, los/las jóvenes de la muestra puede notarse que la mayoría de ellos fue sancionado(a) por delitos de igual o menor gravedad (80\%) acorde a la clasificación del Ministerio Público. De hecho, el $70 \%$ de ellos(as) fue sancionado(a) por el mismo delito por el que ingresó, que en este caso corresponde a delitos contra la propiedad.

CUADRO 7

TASA DE REINCIDENCIA SEGÚN GRAVEDAD DE NUEVO DELITO

\begin{tabular}{|l|c|c|c|c|}
\hline & Nueva Causa & $\%$ & Nueva sanción & $\%$ \\
\hline Igual gravedad & 8 & $27,6 \%$ & 6 & $60 \%$ \\
\hline Menor gravedad & 17 & $58,6 \%$ & 2 & $20 \%$ \\
\hline Mayor gravedad & 4 & $13,8 \%$ & 10 & $20 \%$ \\
\hline Total reincidentes & 29 & & 2 & \\
\hline
\end{tabular}

Elaboración propia.

CUADRO 8

TASA DE REINCIDENCIA SEGÚN TIPO DEL NUEVO DELITO

\begin{tabular}{|l|c|c|c|c|}
\hline & Nueva causa & $\%$ & Nueva sanción & $\%$ \\
\hline Contra propiedad & 13 & $44,8 \%$ & 7 & 0 \\
\hline Porte de armas & 3 & $10,3 \%$ & 3 & $0 \%$ \\
\hline $\begin{array}{l}\text { Contra vida e } \\
\text { integridad física }\end{array}$ & 10 & $34,5 \%$ & 0 & $0 \%$ \\
\hline Drogas & 3 & $10,3 \%$ & 10 & $30 \%$ \\
\hline Total reincidentes & 29 & & 0 & 0 \\
\hline
\end{tabular}

Elaboración propia.

Según puede observarse en el cuadro 9, ninguna de las mujeres que participaron en el programa du- rante ese año habría sido formalizada o sancionada nuevamente dentro del año siguiente a su salida.

CUADRO 9

TASA DE REINCIDENCIA SEGÚN GÉNERO

\begin{tabular}{|l|c|c|c|c|c|}
\hline & Nueva causa & \% nueva causa & Nueva sanción & $\%$ nueva sanción & Total por género \\
\hline Hombre & 29 & $54 \%$ & 10 & $19 \%$ & 54 \\
\hline Mujer & 0 & $0 \%$ & 0 & $0 \%$ & 3 \\
\hline
\end{tabular}

\section{Conclusiones}

Si bien los resultados del programa a la fecha son una mera exploración y de ninguna manera concluyentes o comparables con otros grupos estudiados a nivel nacional o internacional, sí se considera un avance en torno a la cual continuar el trabajo de la Fundación en vías a una futura medición de impacto.

A la fecha, la mayor limitación para desarrollar evaluaciones comprensivas en cuanto al efecto del programa se ha encontrado a la hora de acceder a información de propiedad del Servicio Nacional de
Menores, así como a instrumentos validados para población nacional. Así mismo resulta complejo utilizar instrumentos internacionales en tanto los jóvenes atendidos por el programa, a pesar de cumplir una sanción en el sistema de justicia juvenil, son mayores de 18 años, por lo que no cumplen con los requisitos etarios de instrumentos diseñados para, por ejemplo, medir factores de riesgo en población juvenil.

Pese a esto, los avances en cuanto a la manualización del programa y a la evaluación constante de su ejecución han permitido resolver nudos críticos en 
la implementación y estandarizar procesos y protocolos de acción a seguir, facilitando una ejecución susceptible de evaluar en cuanto a impacto.

\section{Referencias}

BANDURA, A. (1987). Teoría del aprendizaje social. España: Espasa-CALPE.

BARRY, M. (2000) The mentor/monitor debate in criminal justice: 'what works' for offenders. Br J Soc Work 30(5), 575-595

BERNARD, B. (1995). Fostering resilience in children, Clearinghouse on Elementary and Early Childhood Education. Estados Unidos: University of Illinois.

BERRÍOS, G. (2011). La ley de responsabilidad penal del adolescente como sistema de justicia: análisis y propuestas. Polit. Crim. 6(11). 163-191.

BLANCO, J. Y HEIN, A. (2010) Delincuencia juvenil, violencia y desafíos para los programas de intervención. Observatorio, 8. 70-81.

BRONFENBRENNER, U. (1987). La ecología del desarrollo humano. Barcelona: Paidós

BROWN, M. Y ROSS, S. (2010) Mentoring, Social Capital and Desistance: A Study of Women Released from Prison. The Australian and New Zealand Journal of Criminology, 43(1-2)31-50.

BURNETT, R. (2004) To Reoffend or Not to Reoffend? The Ambivalence of Convicted Property Offenders, En Maruna, S. e Immarigeon, R. (eds) After Crime and Punishment: Pathways to Offender Reintegration. Pp 152-180. Devon, Inglaterra y Gales: Willan.

BURNETT, R. Y MARUNA, S. (2004). So Prison Works, Does It? The Criminal Careers of 130 Men Released from Prison under Home Secretary, Michael Howard. Howard Journal 33(4)390-404.

BURNETT, R. Y MARUNA, S. (2006). The kindness of prisoners: Strengths-based resettlement in theory and in action. Criminology and Criminal Justice 6(83).

CASAS, J. Y GONZALEZ, M. (2005) Desarrollo del adolescente. Aspectos físicos, psicológicos y sociales. Pediatr Integral 9(1) 20-24.

CATALANO, R., KOSTERMAN, R., HAWKINS, J., NEWCOMB, M. \& ABBOTT, R. (1996). Modeling the etiology of adolescent substance use: A test of the social development model. Journal of Drug Issues, 26(2), 429-455.

CATALANO. R. \& HAWKINS, D. (1996). The social development model: a theory of antisocial behavior. En J. D. Hawknis Ed. (1996). Delinquency and Crime. Current Theories. Pp.149-197. Australia: Cambridge University Press.

CENTRO DE ESTUDIOS PÚBLICOS EN SEGURIDAD CIUDADANA. (2012) Estudio Modelo y Medición de la Reincidencia de Adolescentes y Jóvenes infractores de la Ley Penal.

CHOI, Y., HARACHI, T., ROGERS, M. \& CATALANO, R. (2005). Applicability of the social development model to urban ethnic minority youth: Examining the relationship between external constrains, family socialization and problem behaviors. Journal of Research on Adolescence, 15(4), 505-534.

COMISIÓN CONSTITUCIÓN, LEGISLACIÓN, JUSTICIA Y REGLAMENTO DEL SENADO CHILENO (2013) Informe de la Comisión de Constitución, Legislación, Justicia y Reglamento, recaído en la evaluación de la aplicación de la ley $\mathrm{N}^{\circ} 20.084$.

COMISIÓN SISTEMA NACIONAL DE CERTIFICACIÓN DE COMPETENCIAS LABORALES [CHILE VALORA] (2013) Capital Humano [Documento en línea]. Recuperado de http://www.chilevalora.cl/index. php/human-capital

DROPPELMANN, C. (2010). Elementos clave en la rehabilitación y reinserción de infractores de ley en Chile. Fundación Paz Ciudadana. Recuperado de http://www. pazciudadana.cl/wp-content/uploads/2010/03/conceptos-14-elementos-clave.pdf

FARRALL, S. (2002). Rethinking What Works with Offenders: Probation, Social Context and Desistance From Crime. Devon, Inglaterra y Gales: Willan.

FARRALL, S. (2004). Social Capital and Offender Reintegration: Making Probation Desistance Focused. En, Maruna S. y Immarigeon, R. (eds) After Crime and Punishment: Pathways to Offender Reintegration. Pp. 57-84. Devon, Inglaterra y Gales: Willan Publishing.

FARRALL, S. Y BOWLING, B. (1999). Structuration, Human Development and Desistance From Crime. British Journal of Criminology 39(2): 252-267.

FARRINGTON, D., COID, J., HARNETT, L., JOLLIFFE, D., SOTERIOU, N., TURNER, R. Y WEST, D. (2006). Criminal careers and life success: new findings from the Cambridge Study in Delinquent Development.

FUNDACIÓN CHILE. (2011). Manual preparado. Programa de Competencias para el Trabajo. Edición de Fundación Chile.

FUNDACIÓN PAZ CIUDADANA. (2010). Construcción de indicadores de reinserción social de adolescentes infractores de la ley penal.

FUNDACIÓN PAZ CIUDADANA (2014) Estrategia en seguridad 2030: Diagnóstico del estado del arte. Documento no publicado.

GRAÑA, J. Y RODRÍGUEZ, J. (2010). Tratamiento educativo y terapéutico para menores infractores. Agencia de la comunidad de Madrid para la reeducación y reinserción del menor infractor.

HAGAN, J. Y MCCARTHY, B. (1997) Mean Streets. Cambridge: Cambridge University Press. 
HEIN, A. (2004). Factores de riesgo y delincuencia juvenil: Revisión de la literatura nacional. Recuperado de http://www.pazciudadana.cl/publs_interior.php?idPublicacion $=68$

HENRY, J. Y JACOBS, J. (2007) Ban the box to promote ex-offender employment. Estados Unidos: Proyecto para Leyes Laborales Nacionales (National Employment Law Project [NELP]). Recuperado de http://www. nelp.org/page/-/SCLP/Henry-Jacobs.BantheBox.article. Oct-07

HIRSCHI, TRAVIS. (1969). Causas de la delincuencia. Berkeley y Los Angeles: University of California Press.

KAZEMIAN, L. Y MARUNA, S. (2009) Desistance from Crime en Khron, M., Lizotte, A. y Penly, G. Ed. (2009) Handbook on Crime and Deviance. Estados Unidos: Springer.

KC4ALL PROJECT TEAM. (2011) Key Competences For All [KC4ALL] (Kit de Herramientas para la Empleabilidad): Manual para facilitadores y capacitadores. Recuperado de http://www.keycompetences.eu/ wordpress/spanish-toolkit/files/2011/10/handbook_final-Edited-08-08-2011-v1-ES.pdf

LAUB, J. Y SAMPSON, R. (2001). Understanding desistance from crime. Crime and Justice 28.

LIPSEY, M. WILSON B. Y COTHERN, L. (2000) Effective Intervention for Serious Juvenile Offenders. Juvenile Justice Bulletin. Estados Unidos: Office of Juvenile Justice and Delinquency Prevention Recuperado de https://www.ncjrs.gov/pdffiles1/ojjdp/181201.pdf

LIPSEY, M. Y CULLEN, F. (2007). The Effectiveness Of Correctional Rehabilitation: A Review Of Systematic Reviews. Annual Review of Law and Social Science 3.

LONCZAK, H., HUANG, B., CATALANO, R., HAWKINS, J., HILL, K., ABBOTT, R., RYAN, J. \& KOSTERMAN R. (2001) The social predictors of adolescent alcohol misuse: a test of the social development model. Journal of Studies on Alcohol and Drugs, 62(2),179-89.

MACKENZIE, D. (2006). What Works in Corrections: Reducing the Criminal Activities of Offenders and Delinquents. Estados Unidos: Cambridge University Press.

MAGUIRE, M. Y RAYNOR, P. (2006) How the resettlement of prisoners promotes desistance from crime Or does it? Criminology and Criminal Justice 6 (1) pp. 19-38

MARLER, L. (2008). Proactive Behavior: A Selection Perspective. Estados Unidos: Universidad de Louisiana.

MARTÍN, M. Y SPINOSA, A. (2005). Del empleo a la empleabilidad, de la educación a la educabilidad. Mutaciones conceptuales e individualización de los conflictos sociales. Séptimo Congreso de Especialista en estudios del Trabajo. Grupo 10: Educación y Mercado de Trabajo.

MARTINIC, S. Y WALKER, H. (1987). La Reflexión Metodológica en el Proceso de Sistematización de Experiencias de Educación Popular. En Cadena, Felix y otros (1987).
La Sistematización en los Proyectos de Educación Popular. Santiago, Chile: Edición CEAAL.

MARUNA, S. (1999). Desistance and Development: The Psychosocial Process of Going Straight. British Society of Criminology Proceedings 2, p. 1-25.

MARUNA, S. (2001). Making Good: How Ex-Convicts Reform and Rebuild Their Lives. Washington D.C, Estados Unidos: American Psychological Association Books.

MARUNA, S. (2012) A Signaling Perspective on Employment-Based Reentry: Elements of Successful Desistance Signaling. Criminology \& Public Policy 11(1)

MARUNA, S. Y FARRALL, S. (2004) Desistance Focused Criminal Justice Policy Research: Introduction to a Special Issue on Desistance from Crime and Public Policy. The Howard Journal of Criminal Justice 43 (4), 358-367.

MCNEILL, F. (2006). A desistance paradigm for offender management. Criminology and Criminal Justice, 6, 39-62.

MEARS, D. Y TRAVIS, J. (2004). The Dimensions, Pathways, and Consequences of Youth Reentry. Estados Unidos: Urban Institute. Recuperado de http:// www.urban.org/publications/410927.html

MERTZ, C. (2013) Delincuencia en Chile: diagnóstico y propuestas. En Grupo Res (2013) 95 Propuestas para un Chile Mejor. Recuperado de http://95propuestas. cl/site/wp-content/uploads/2013/05/delincuencia-enchile-diagnostico-y-propuestas-catalina-mertz.pdf

MILlER, J. (200) 21st Century Criminology: A Reference Handbook. SAGE publications.

MONAHAN, K., STEINBERG, L., \& CAUFFMAN, E. (2013). Age differences in the impact of employment on antisocial behavior. Child Development, 84.

MORALES, A., WELSCH, G., CÁRCAMO, J., AGUILAR, L. Y SOSA, M.E. (2013) Reinserción Social y Laboral: Estudio y comparado de la evidencia internacional y nacional existente en torno a la reinserción social de los infractores de ley, con enfoque laboral, en adultos y jóvenes.

MULVEY, E. (2011) Highlights From Pathways to Desistance: A Longitudinal Study of Serious Adolescent Offenders. Juvenile Justice Bulletin. Estados Unidos: Ministerio de Justicia. Oficina de Prevención del delito y Justicia Juvenil (Office of Juvenile Justice and Delinquency Prevention).

NAGIN, D. Y WALDFOGEL, J. (1998). The Effect of Conviction on Income Through the Life Cycle. International Review of Law and Economics, Elsevier 18(1), pages 25-40.

NATIONAL INSTITUTE OF CORRECTIONS [NIC]. (2011). Thinking for a Change: Integrated Cognitive Behavior Change Program. Version 3.1.

O'DONELL, I., BAUMER, E. \& HUGHES, N. (2008). Recidivism in the Republic of Ireland. Criminology and Criminal Justice, 8(2). 
OFICINA INTERNACIONAL DEL TRABAJO [OIT] (2012). Impulso a la empleabilidad de jóvenes desfavorecidos.

ORTEGO, M., LÓPEZ, S., ÁlVAREZ, L. (2010). Material del curso Ciencias Psicosociales I. Tema: La comunicación. Universidad de Cantabria.

PRZYBYLSKI, R. (2008) What Works: Effective Recidivism Reduction and Risk-Focused Prevention Programs. A Compendium of Evidence-Based Options for Preventing New and Persistent Criminal Behavior. RKC Group. Estados Unidos: División de Justicia Criminal (Division of Criminal Justice).

SAMPSON, R. Y LAUB, J. (1993). Crime in the Making: Pathways and Turning Points through Life. Estados Unidos: Harvard University Press.

SANCHEZ-TERUEL, D. (2012). Factores de riesgo y protección ante la delincuencia en menores y jóvenes. Revista social 15.

SEITER, R. (2004). Inmate Reentry: What Works and What to do About it. Corrections Today 64(2).

SERVICIO NACIONAL DE MENORES [SENAME]. (2012). Informe 5 años de LRPA. Recuperado de http:// www.SENAME.cl/wSENAME/otros/INFORME_ ESTADISTICO_5A_LRPA.pdf.

SHERMAN, L.W., FARRINGTON, D., WELSH, B. Y MACKENZIE, D. (2002) Evidence-Bases Crime Prevention. London: Routledge.

SOCIAL EXCLUSION UNIT. (2002). Reducing re-offending by ex-prisoners. Reino Unido: Office of the Deputy Prime Minister.

STEINBERG, L., CHUNG, H. L. Y LITTLE, M. (2004). Reentry of Young Offenders From the Justice System: A Developmental Perspective. Youth Violence Juv Justice 2(1).

STEPHENSON, M., GILLER, H. Y BROWN, S. (2007). Effective Practice y Youth Justice. Londres: Willan Publishing.

TANNER, J., DAVIES, S. Y O'GRADY, B. (1999). Whatever happened to yesterday's rebels? Longitudinal effects of youth delinquency on educational employment. Social Problems 46, 250-274.

TENNYSON, H. (2009) Reducing Juvenile Recidivism: A Meta-Analysis of Treatment Outcomes. School of Professional Psychology 109

UGGEN, C. (1999). Ex-Offenders and the Conformist Alternative: A Job Quality Model of Work and Crime. Social Problems 46(1) 127-151.

UGGEN, C. Y WAKEFIELD, S. (2003). Young Adults Reentering The Community From The Criminal Justice System: The Challenge Of Becoming An Adult. [Borrador preparado para] On Your Own Without A Net: The Transition To Adulthood For Vulnerable Populations. Osgood, W., Foster, M., Flanagan, C. y Gretchen, R. [Ed.]. (2005). Estados Unidos: University Of Chicago Press.
UMATERR PROJECT. (2013). Detour Program. Recuperado de http://thedetourprogram.org/the-detour-programs/

VALDENEGRO, B. (2005). Factores psicosociales asociados a la delincuencia juvenil. Psykhe, 14(2), 33-42.

VILLAGRA, C. (2007). Hacia una política postpenitenciaria en Chile. Centro de Estudios en Seguridad Ciudadana. Santiago de Chile: Ril Editores.

VISHER, C. DEBUS, S. Y YAHNER, J. (2008). Employment after Prison: A Longitudinal Study of Releasees in Three States. Estados Unidos: Urban institute, Justice Policy Center.

WALDFOGEL, J. (1994). Does conviction have a persistent effect on income and employment? International Review of Law and Economics, Elsevier, 14(1), pages 103119.

WALKER, G. (1997). A Generation of Challenge: Pathways to Success for Urban Youth. Levitan Youth Policy Network. Policy Issues Monograph 97-03.

WILSON, D., GALlAGHER, C. Y MACKENZIE, D. (2000) A Meta-Analysis of Corrections-Based Education, Vocation, and Work Programs for Adult Offenders. Journal of Research in Crime and Delinquency, 37(4) $347-368$ 
